\title{
ALTERAÇÕES FÍSICAS E QUÍMICAS DURANTE O DESENVOLVIMENTO DE MANGAS 'TOMMY ATKINS' NO VALE DO SÃO FRANCISCO, PETROLINA-PE ${ }^{1}$
}

\author{
ELISEU MARLÔNIO PEREIRA DE LUCENA², JOSTON SIMÃO DE ASSIS ${ }^{3}$, RICARDO ELESBÃO ALVES ${ }^{4}$, \\ VÍCTOR CÉSAR MACÊDO DA SILVA 5 , JOAQUIM ENÉAS FILHO ${ }^{6}$
}

RESUMO - O presente trabalho objetivou caracterizar o crescimento dos frutos de mangueira (Mangifera indica L.), cv. Tommy Atkins, da antese até a colheita comercial, visando à definição do ponto de colheita ideal baseado em determinações físicas e físicoquímicas. Os frutos foram colhidos aos 35; 49; 63; 70; 77; 84; 98 e 112 dias após a antese (DAA), sendo feitas as seguintes determinações: diâmetro longitudinal, diâmetro ventral, diâmetro transversal, produto dos diâmetros, massa fresca, massa seca, massa e teor de água, escala de Blush para coloração da casca e sólidos solúveis. O trabalho indicou que as mangas atingiram a maturidade aos 98 DAA e que a massa seca, dentre os estudados, é o melhor indicador do estádio de desenvolvimento dos frutos. Termos para indexação: Mangifera indica, fisiologia pós-colheita, crescimento do fruto, Tommy Atkins.

\section{PHYSICAL AND CHEMICAL CHANGES DURING TOMMY ATKINS MANGOES DEVELOPMENT IN SÃO FRANCISCO VALLEY, PETROLINA, PE, BRAZIL}

\begin{abstract}
The objective of this study was to characterize the mango (Mangifera indica L.) fruit growth, cv. Tommy Atkins, from anthesis to commercial harvest, identifying when the mango reaches the harvest maturity stage based on physical and physicochemical determinations. The fruits were harvested at 35, 49, 63, 70, 77, 84, 98 and 112 days after the anthesis (DAA), and the following determinations were made: longitudinal diameter, ventral diameter, transversal diameter, product of the diameters, fresh mass, dry mass, water content and percentage, Blush scale for skin color and soluble solids. This study indicated that mango cv. Tommy Atkins delayed 98 DAA to reach the maturity and the dry mass, among those studied, is the best growth indicator.
\end{abstract}

Index terms: Mangifera indica, postharvest physiology, fruit growth, Tommy Atkins.

\section{INTRODUÇÃO}

De acordo com Almeida et al. (2005), a mangicultura é uma das principais atividades do agronegócio frutícola do Brasil, apresentando desempenho crescente nos últimos anos.É o nono maior produtor mundial de manga, onde a Índia é o primeiro, e desde 1999 o segundo maior exportador dessa fruta, perdendo apenas para o México. O Brasil vem ampliando sua participação nas exportações mundiais e gerando empregos e renda em todo o território nacional, especialmente no Nordeste, a região que mais exporta manga, destacando-se os Estados da Bahia e Pernambuco.

A cultivar Tommy Atkins produz frutos classificados botanicamente como drupa, de tamanho médio para grande (400 a $700 \mathrm{~g}$ ), formato ovalado a oblongo, superfície lisa, com casca espessa, e coloração laranja-amarela coberta com vermelho e púrpura intensa. A polpa apresenta cor amarelo-escura, consistência firme, suculenta e contendo teor médio de fibras. No interior da polpa, encontra-se a semente monoembriônica, pequena, representando de 6 a $8 \%$ do peso do fruto, que é recoberta por um endocarpo fibroso (Cunha et al. 1994; Donadio,

\section{2; Pinto et al., 2002).}

Todos os aspectos do crescimento e desenvolvimento da mangueira são direta ou indiretamente influenciados pelo ambiente. Assim, o conhecimento das respostas desta planta às variações ambientais é de fundamental importância para o estabelecimento de um manejo adequado às condições correntes, visando à melhoria da qualidade dos frutos e à maximização da produtividade.

Segundo Cunha et al. (2002), a época de maturação dos frutos, cujo crescimento apresenta padrão sigmóide, varia entre as diversas regiões produtoras, e o período de seu desenvolvimento (da floração à maturidade) é, em geral, de $100 \mathrm{a}$ 150 dias. Em regiões mais quentes, esse período é menor.

A fim de dar uma resposta à falta de informação sobre como o fruto de manga 'Tommy Atkins' cresce, e quais os fatores que afetam o seu desenvolvimento, foi realizada a presente pesquisa, que objetivou caracterizar o desenvolvimento da antese até a colheita comercial, de frutos de mangueira (Mangifera indica L.), cv. Tommy Atkins, cultivada no Vale do São Francisco, visando à definição do ponto de colheita ideal baseado em determinações físicas e físico-química.

\footnotetext{
${ }^{1}$ (Trabalho 022-2006). Recebido em 07-03-2006. Aceito para publicação em 05-12-2006.

${ }^{2}$ Universidade Estadual do Ceará - UECE, Ciências Biológicas, Av. Paranjana, 1700, Itaperi, CEP 60.740-903, Fortaleza-CE, Brasil, eliseulucena@uece.br

3 Embrapa Semi-Árido, CEP 56302-970, Petrolina-PE, Brasil, joston@ cpatsa.embrapa.br

${ }^{4}$ Embrapa Agroindústria Tropical, CEP 60511-110, Fortaleza-CE, Brasil, elesbao@ cnpat.embrapa.br

${ }^{5}$ Universidade de Pernambuco - UPE, vcmbahia@yahoo.com.br

${ }^{6}$ Universidade Federal do Ceará - UFC, Departamento de Bioquímica e Biologia Molecular, Campus do Pici, B1. 907, CEP 60455-760, Fortaleza-CE, Brasil, joaquime@ufc.br
} 


\section{MATERIAL E MÉTODOS}

Este trabalho foi conduzido na Fazenda Fruit Fort Agrícola Exportação Ltda. e no Laboratório de Fisiologia Pós-Colheita da Embrapa Semi-Árido, no município de Petrolina- PE, durante o período de junho a dezembro de 2004.

O clima da região é do tipo BSwh', segundo a classificação de Köeppen, que corresponde a uma região quente e semi-árida. A área de plantio apresenta as seguintes características: latitude $9^{\circ} 22^{\prime} \mathrm{S}$, longitude $40^{\circ} 33^{\prime} \mathrm{W}$, altitude $388 \mathrm{~m}$, temperatura média $26,05^{\circ} \mathrm{C}$, com médias máximas de $32,03^{\circ} \mathrm{C}$ e médias mínimas de $20,38^{\circ} \mathrm{C}$, umidade média mensal é de $66,25 \%$, radiação solar global média de 20,94 MJ/m²/dia, a velocidade média dos ventos é de $2,05 \mathrm{~m} / \mathrm{s}$ com direções $\mathrm{E}$ e $\mathrm{S}$ e a precipitação pluvial média anual é de 535,53 mm; o solo é classificado como Latossolo VermelhoAmarelo-LV (Embrapa, 2006).

Nas Figuras 1 e 2, encontram-se as características climáticas médias ou totais referentes ao ano que antecedeu as colheitas, de acordo com os dados fornecidos pelo Centro de Pesquisa Agropecuária do Tropico Semi-Árido - Embrapa. Os dados foram coletados a partir da Estação Meteorológica Automatizada na Fazenda Fruit Fort Agrícola Exportação Ltda.

No início do ciclo, em novembro de 2003, foram feitas análises de fertilidade do solo da rizosfera e foliar das mangueiras. Os resultados destas análises encontram-se listadas na Tabela 1. De posse dos resultados das análises, foram incorporados na adubação nitrogênio (nitrato de cálcio-418,3 g/planta e uréia0,53 g/planta), fósforo (MAP-571,15 g/planta, ácido fosfórico0,061 g/planta e nutriox P30-9,47 mL/planta) e potássio (cloreto de potássio-0,61 g/planta, sulfato de potássio-682 g/planta e nitrato de potássio-1.067 g/planta). O magnésio foi incorporado na forma de sulfato de magnésio (228,03 g/planta). Os micronutrientes ( $\mathrm{Zn}, \mathrm{Fe}, \mathrm{B}$ e $\mathrm{Mn}$ ) também foram incorporados. Os macro e micronutrientes foram aplicados via pulverização e fertirrigação em 16 e 6, parcelas respectivamente, distribuídas eqüitativamente durante todo o ciclo da cultura.

Mangueiras da cv. Tommy Atkins enxertadas sobre a cv. Espada, com 21 anos de idade, foram submetidas a indução floral, de acordo com as recomendações técnicas (Albuquerque et al., 2002). O lote media 6,09 ha. Era composto de 655 plantas, irrigadas por microaspersão, com turno de rega diário, iniciando após a indução floral com uma lâmina bruta de 89 litros/planta, sendo ao longo do ciclo ajustado em função das características climáticas registradas. As mangueiras possuíam altura média de $5,5 \mathrm{~m} \mathrm{e}$ estavam dispostas no espaçamento 10 x $10 \mathrm{~m}$. O controle de plantas invasoras foi realizado mensalmente, através de capinas com enxada e roço com estrovenga ou com roçadeira acoplada ao trator.

Durante a antese, isto é, quando $65 \%$ das plantas haviam emitido panículas, foram selecionadas 211 plantas, onde 797 panículas foram marcadas com fitas de plástico e monitoradas até 112 dias após a antese (DAA). O delineamento experimental utilizado foi o inteiramente casualizado, com 8 tratamentos (épocas de colheita) e 3 repetições, constituídas por quatro frutos cada.

Antes da colheita, os frutos foram individualmente numerados. Colheram-se 24 frutos aos $35 ; 49 ; 63 ; 70 ; 77 ; 84 ; 98$ e 112 DAA de outubro a dezembro de 2004. Após a colheita, os frutos foram transportados para o laboratório e imediatamente pesados, individualmente, em balança semi-analítica para a obtenção da massa fresca (MF). Esse processo, da colheita até o final das pesagens, foi efetuado em, no máximo, 60 minutos. Esse tempo foi rigorosamente mantido para minimizar as perdas de água e variações entre as amostragens.

Para a obtenção da massa seca (MS), os frutos inteiros foram fracionados em pequenos pedaços e colocados para secar em estufa de circulação forçada de ar a $60^{\circ} \mathrm{C}$, até a obtenção de peso constante em balança semi-analítica. O tempo de secagem variou de 24 a 72 horas, de acordo com o tamanho do fruto. Após a secagem, o material foi colocado em dessecador contendo sílica gel para esfriar de forma a minimizar a sua reidratação antes da pesagem.

A massa de água do fruto, expressa em gramas, foi determinada por diferença entre a MF e a MS. O teor de água no fruto, expresso em percentagem, foi determinado através da fórmula: teor de água = massa de água $\mathrm{x} \mathrm{MF}^{-1} \mathrm{x} 100$.

$O$ volume dos frutos foi calculado individualmente através do produto entre os diâmetros longitudinal, ventral e transversal (Castro Neto \& Reinhardt, 2003), determinados mediante o uso de paquímetro digital. Os resultados do produto dos diâmetros foram expressos em centímetros cúbicos $\left(\mathrm{cm}^{3}\right)$.

A escala de Blush para a coloração da casca é uma medida subjetiva onde é avaliado o percentual de cor vermelha na manga (Amorim, 2002). A escala é enumerada de 1 a 5 , cada número correspondendo a uma faixa percentual da cor vermelha: 1-0\%; 2->0-25\%; 3->25-50\%; 4->50-75\%; e 5->75-100\%. Os resultados foram expressos em média de notas atribuídas por repetição, através do exame individual de cada manga por quatro avaliadores treinados.

Os sólidos solúveis foram obtidos através de um refratômetro digital Atago modelo PR-101, escala de 0 a $45^{\circ}$ Brix, com compensação de temperatura automática (AOAC, 1995).

Os dados obtidos em função do tempo de amostragem foram submetidos à análise de variância, observando a significância pelo teste F. Para os casos em que os tratamentos foram significativos, procederam-se a ajustes através de regressões polinomiais. O Software ESTAT (Sistema para Análises Estatísticas) foi utilizado nestes cálculos. Além disso, foram estimados os coeficientes de correlação linear entre as variáveis estudadas, verificando-se a significância pelo teste t, através do programa estatístico ASSISTAT (Assistência Estatística).

\section{RESULTADOS E DISCUSSÃO}

Os efeitos fixos de tratamentos para massa fresca, massa seca e teor de água são explicados em $90 \%$ pela equação de regressão cúbica $\left(R^{2}=0,92, R^{2}=0,99\right.$ e $R^{2}=0,93$ respectivamente), enquanto, para a massa de água, $85 \%$ pela equação de regressão quadrática (Figura 3).

Podemos observar ainda, na Figura 3, que o acúmulo da massa seca ocorreu até 98 dias após a antese (DAA), coincidindo 


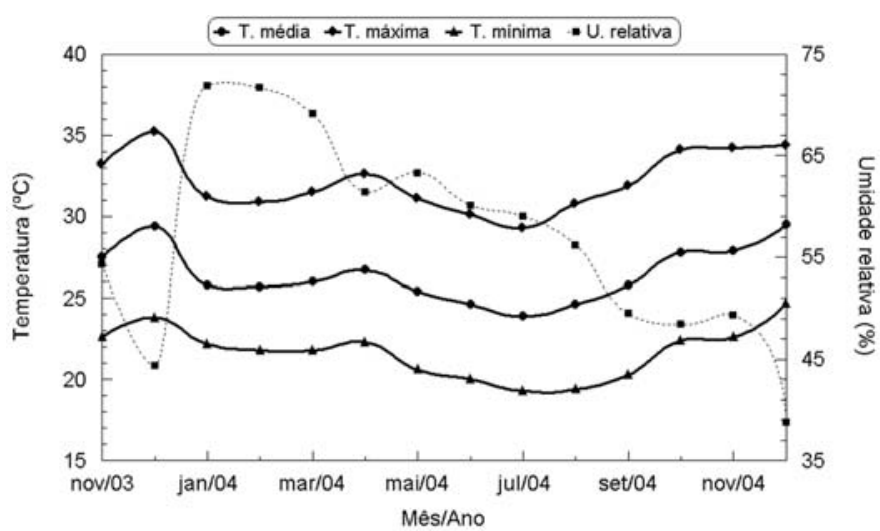

FIGURA 1 - Temperaturas e umidade relativa do ar na região de Petrolina - PE, durante o ano que antecedeu as colheitas (outubro a dezembro/04) das mangas cv. Tommy Atkins.



FIGURA 2 - Radiação solar global e precipitação pluviométrica na região de Petrolina - PE, durante o ano que antecedeu as colheitas (outubro a dezembro/04) das mangas cv. Tommy Atkins.

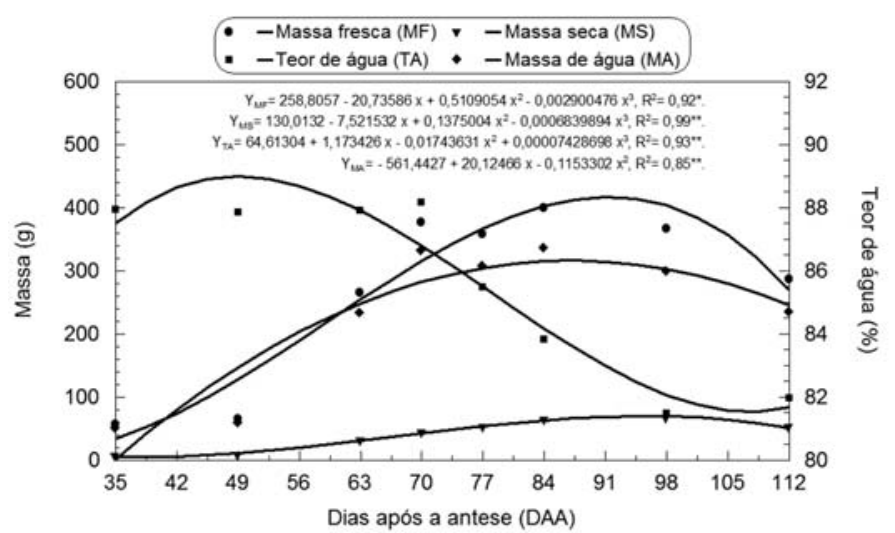

FIGURA 3 - Evolução das massas fresca e seca, teor e massa de água durante o desenvolvimento do fruto da cv. Tommy Atkins, a partir da antese. com a mudança da coloração da casca, na escala Blush, de 3 para 4 (Figura 6). O percentual da cor vermelha evoluiu da faixa de $>25-50 \%$ para $>50-75 \%$. Isto caracteriza o início da maturação do fruto, quando simultaneamente se registrou o menor teor de água. A diferença de acúmulo da massa seca entre 84 e 98 DAA foi de apenas 3,28 g. Esse conjunto de resultados sugere que, para as condições estudadas, a manga cv. Tommy Atkins atinge a maturação aos 98 DAA.

O período registrado neste trabalho (98 DAA) foi superior aos 75 dias para a cv. Haden atingir o ponto de colheita (Castro Neto \& Reinhardt, 2003), na mesma região, e aos 90 dias relatados para outras cultivares, como Langra, Alphonso, Dashehari e Krishnabhog, na Índia (Subramanyan et al., 1975). Essas diferenças podem ser atribuídas a variações no ciclo de crescimento das plantas e, conseqüentemente, na duração do período reprodutivo e de crescimento do fruto. No entanto, Morais (2001), estudando o ponto de maturidade para colheita e a vida útil da manga 'Tommy Atkins' para o mercado Europeu, cultivada no Pólo Mossoró-Assu, Rio Grande do Norte, constatou que, 96 dias após a floração plena, pode ser considerado como um indicador do ponto de colheita.

A taxa de acúmulo da massa seca no fruto foi maior no período de 49 a 98 DAA. Conforme Castro Neto \& Reinhardt (2003), o acúmulo da massa seca pode ser conseqüência de maior participação da fotossíntese no período, associada à translocação da matéria seca de outras partes da planta para o fruto, sendo que a remobilização de fotoassimilados previamente fotossintetizados é de $40-50 \%$ do peso seco do fruto.

Os valores máximos da massa fresca e massa de água do fruto foram, respectivamente, 400,00 g e 335,66 g, aos 84 DAA, havendo em seguida decréscimo, associado à redução no conteúdo de água do fruto, corroborando, assim, os resultados de Castro Neto \& Reinhardt (2003), que, estudando o crescimento de frutos, cv. Haden, constataram que os valores da massa fresca e massa de água máximos foram atingidos antes de chegar à maturidade, isto é, quando a massa seca foi máxima.

Na Figura 4, verifica-se que os diâmetros longitudinal, ventral e transversal apresentaram comportamento quadrático em resposta à variação de tempo, onde as mangas aumentaram em tamanho até 70 DAA, quando se avalia o diâmetro longitudinal, e até 77 DAA, quando se consideram os diâmetros ventral e transversal, e após estes períodos praticamente se mantêm constante. Neste sentido, Leite et al. (2005), pesquisando as características físicas da manga cv. Tommy Atkins, produzida na região do Vale do São Francisco, município de Petrolina-PE, no período de setembro a outubro de 2004, obtiveram os seguintes valores médios, respectivamente, para diâmetros longitudinal, ventral e transversal: 10,89; 9,55 e 8,67 cm. Esses valores são da mesma ordem de grandeza dos obtidos neste trabalho.

A Figura 5 indica que o produto dos diâmetros apresentou comportamento quadrático em resposta à variação de tempo. Conforme Morais (2001) e Castro Neto \& Reinhardt (2003), podese estimar, com precisão, as massas fresca e seca das mangas cvs. Tommy Atkins e Haden, respectivamente, a partir do produto dos diâmetros. 
TABELA 1 - Análises de fertilidade do solo da rizosfera e de tecido foliar de mangueiras cv. Tommy Atkins, no início do ciclo da cultura, em novembro de 2003.

\begin{tabular}{cccc}
\hline Determinações & Solo $(0-20 \mathrm{~cm})$ & Solo $(20-40 \mathrm{~cm})$ & Folha \\
\hline N & - & - & $12,10 \mathrm{~g} / \mathrm{kg}$ \\
M.O. & $8,00 \mathrm{~g} / \mathrm{dm}^{3}$ & $2,00 \mathrm{~g} / \mathrm{dm}^{3}$ & - \\
pH SMP & 7,40 & 7,40 & - \\
pH & 6,20 & 6,20 & $1,20 \mathrm{~g} / \mathrm{kg}$ \\
$\mathrm{P}$ & $23,00 \mathrm{mg} / \mathrm{dm}^{3}$ & $12,00 \mathrm{mg} / \mathrm{dm}^{3}$ & $7,30 \mathrm{~g} / \mathrm{kg}$ \\
$\mathrm{K}$ & $1,30 \mathrm{mmolc} / \mathrm{dm}^{3}$ & $1,20 \mathrm{mmolc} / \mathrm{dm}^{3}$ & $20,30 \mathrm{~g} / \mathrm{kg}$ \\
$\mathrm{Ca}^{2+}$ & $30,00 \mathrm{mmolc} / \mathrm{dm}^{3}$ & $18,00 \mathrm{mmolc} / \mathrm{dm}^{3}$ & $1,10 \mathrm{~g} / \mathrm{kg}$ \\
$\mathrm{Mg}^{2+}$ & $4,00 \mathrm{mmolc} / \mathrm{dm}^{3}$ & $3,00 \mathrm{mmolc} / \mathrm{dm}^{3}$ & $185,00 \mathrm{mg} / \mathrm{kg}$ \\
$\mathrm{Na}$ & $0,20 \mathrm{mmolc} / \mathrm{dm}^{3}$ & $0,20 \mathrm{mmolc} / \mathrm{dm}^{3}$ & $280,00 \mathrm{mg} / \mathrm{kg}$ \\
$\mathrm{Al}$ & $<1,00 \mathrm{mmolc} / \mathrm{dm}^{3}$ & $<1,00 \mathrm{mmolc} / \mathrm{dm}^{3}$ & - \\
$\mathrm{H}$ & $9,70 \mathrm{mmolc} / \mathrm{dm}^{3}$ & $9,70 \mathrm{mmolc} / \mathrm{dm}^{3}$ & - \\
$\mathrm{H}+\mathrm{Al}$ & $10,00 \mathrm{mmolc} / \mathrm{dm}^{3}$ & $10,00 \mathrm{mmolc} / \mathrm{dm}^{3}$ & - \\
$\mathrm{S} . \mathrm{B}$. & $35,30 \mathrm{mmolc} / \mathrm{dm}^{3}$ & $22,20 \mathrm{mmolc} / \mathrm{dm}^{3}$ & - \\
$\mathrm{C} . \mathrm{C}$. & $45,00 \mathrm{mmolc} / \mathrm{dm}^{3}$ & $31,90 \mathrm{mmolc} / \mathrm{dm}^{3}$ & - \\
$\mathrm{V}$ & $78,00 \%$ & $70,00 \%$ & $1,30 \mathrm{~g} / \mathrm{kg}$ \\
$\mathrm{S}$ & $4,00 \mathrm{mg} / \mathrm{dm}^{3}$ & $2,00 \mathrm{mg} / \mathrm{dm}^{3}$ & $97,86 \mathrm{mg} / \mathrm{kg}$ \\
$\mathrm{B}$ & $0,33 \mathrm{mg} / \mathrm{dm}^{3}$ & $0,29 \mathrm{mg} / \mathrm{dm}^{3}$ & $28,00 \mathrm{mg} / \mathrm{kg}$ \\
$\mathrm{Cu}$ & $10,30 \mathrm{mg} / \mathrm{dm}^{3}$ & $1,90 \mathrm{mg} / \mathrm{dm}^{3}$ & $175,00 \mathrm{mg} / \mathrm{kg}$ \\
$\mathrm{Fe}$ & $14,00 \mathrm{mg} / \mathrm{dm}^{3}$ & $34,00 \mathrm{mg} / \mathrm{dm}^{3}$ & $275,00 \mathrm{mg} / \mathrm{kg}$ \\
$\mathrm{Mn}$ & $34,50 \mathrm{mg} / \mathrm{dm}^{3}$ & $16,50 \mathrm{mg} / \mathrm{dm}^{3}$ & $17,00 \mathrm{mg} / \mathrm{kg}$ \\
\hline $\mathrm{Zn}$ & $145,80 \mathrm{mg} / \mathrm{dm}^{3}$ & $285,80 \mathrm{mg} / \mathrm{dm}^{3}$ & \\
\hline
\end{tabular}

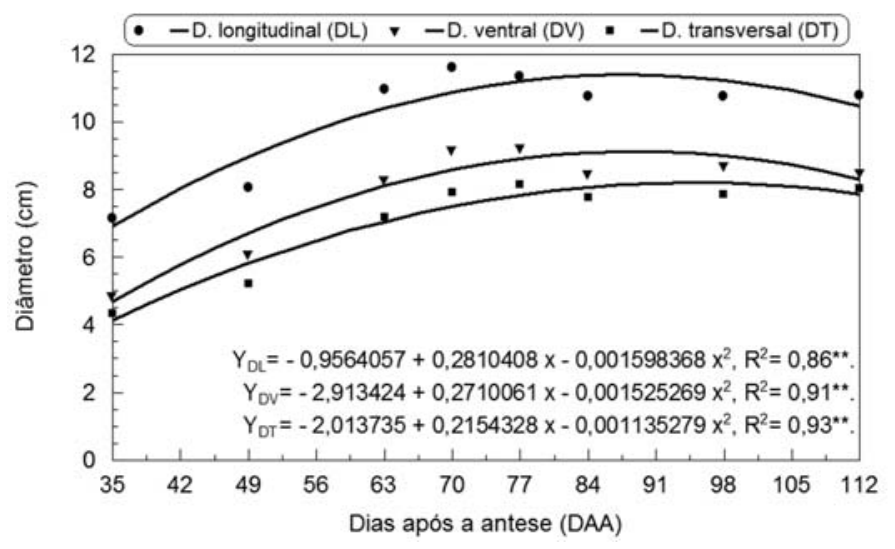

FIGURA 4 - Evolução dos diâmetros longitudinal, ventral e transversal durante o desenvolvimento do fruto da cv. Tommy Atkins, a partir da antese.



FIGURA 5 - Evolução do produto dos diâmetros durante o desenvolvimento do fruto da cv. Tommy Atkins, a partir da antese.

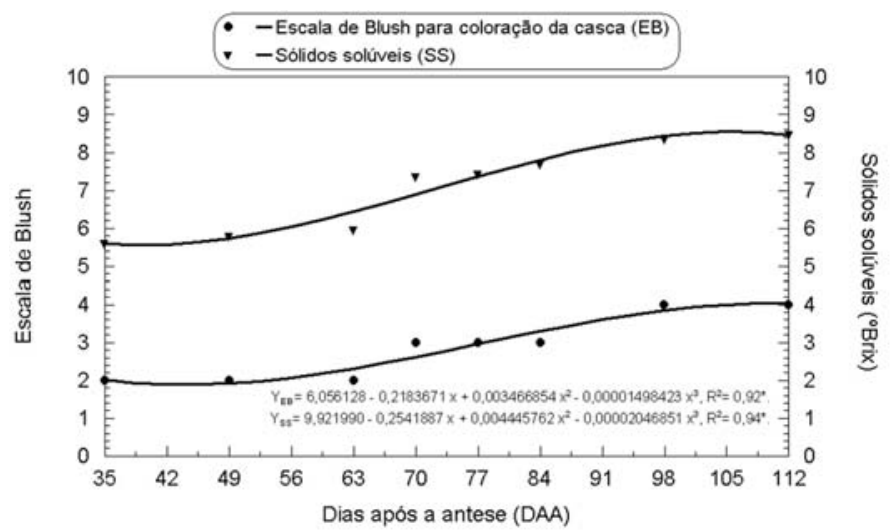

FIGURA 6 - Evolução da escala de Blush para coloração de casca e sólidos solúveis durante o desenvolvimento do fruto da cv. Tommy Atkins, a partir da antese.

Embora nem todos os frutos mudem de cor durante o amadurecimento, essa é uma das características mais associadas ao ponto de colheita e maturidade para consumo (Tucker, 1993). Essa afirmação é particularmente verdadeira na colheita da manga. As avaliações de mudança de coloração da casca, na escala de Blush (Figura 6), mostram claramente essa tendência.

$\mathrm{O}$ aumento no teor de sólidos solúveis durante o desenvolvimento foi contínuo, partindo-se de 5,6 ${ }^{\circ} \mathrm{Brix}$ aos 35 DAA, para 8,5 Brix aos 112 DAA (Figura 6). Lederman et al. (1998) encontraram, para manga 'Tommy Atkins' em idades variando de 95 a 125 DAA, sólidos solúveis variando de 7,5 a 10,9³rix.

Os coeficientes de correlação linear determinados com o intuito de medir o grau de associação entre as variáveis estudadas encontram-se na Tabela 2. Dentre os coeficientes de correlação linear estimados, para todas as combinações possíveis, apenas para o teor de água foram observadas correlações negativas e 
TABELA 2 - Coeficientes de correlação linear estimados (R), entre as variáveis estudadas durante o desenvolvimento do fruto da cv. Tommy Atkins, a partir da antese.

\begin{tabular}{|c|c|c|c|c|c|c|c|c|c|}
\hline Variáveis & $\overline{\mathrm{DV}}$ & DT & PD & MF & MS & MA & TA & EB & SS \\
\hline Diâmetro longitudinal (DL) & $0,96^{* *}$ & $0,94 * *$ & $0,98^{* *}$ & $0,77 * *$ & $0,69 * *$ & $0,77 * *$ & $-0,34^{\mathrm{ns}}$ & $0,41 *$ & $0,60 * *$ \\
\hline Diâmetro ventral (DV) & - & $0,97 * *$ & $0,98 * *$ & $0,80 * *$ & $0,74 * *$ & $0,80 * *$ & $-0,42 *$ & $0,51 *$ & $0,69 * *$ \\
\hline Diâmetro transversal (DT) & - & - & $0,96^{* *}$ & $0,85^{* *}$ & $0,82 * *$ & $0,84 * *$ & $-0,54 * *$ & $0,63 * *$ & $0,78 * *$ \\
\hline Produto dos diâmetros (PD) & - & - & - & $0,79 * *$ & $0,73 * *$ & $0,79 * *$ & $-0,42 *$ & $0,50 *$ & $0,69 * *$ \\
\hline Massa fresca (MF) & - & - & - & - & $0,94 * *$ & $0,99 * *$ & $-0,47^{*}$ & $0,54 * *$ & $0,72 * *$ \\
\hline Massa seca (MS) & - & - & - & - & - & $0,92 * *$ & $-0,71 * *$ & $0,68 * *$ & $0,83 * *$ \\
\hline Massa de água (MA) & - & - & - & - & - & - & $-0,41 *$ & $0,50 *$ & $0,69 * *$ \\
\hline Teor de água (TA) & - & - & - & - & - & - & - & $-0,82 * *$ & $-0,81 * *$ \\
\hline Escala de Blush (EB) & - & - & - & - & - & - & - & - & $0,87 * *$ \\
\hline
\end{tabular}

*, ** Significativos, respectivamente, em nível de 5 e de $1 \%$ de probabilidade, pelo teste t.

ns Não-significativo. SS - Sólidos solúveis.

significativas em relação às demais, com exceção quando correlacionada ao diâmetro longitudinal que, apesar de continuar negativa, foi não- significativa. As demais correlações possíveis foram positivas e significativas. Os resultados da correlação entre o produto dos diâmetros e as massas fresca e seca, apesar de apresentarem menores valores de coeficientes $(\mathrm{R}=0,79$ e $\mathrm{R}=$ $0,73)$, assemelham-se àqueles obtidos por Morais (2001), estudando essa mesma cultivar $(\mathrm{R}=0,97$ e $\mathrm{R}=0,94)$, e Castro Neto \& Reinhardt (2003), estudando a cv. Haden ( $R=0,99$ e $R=$ $0,95)$.

Apesar de a massa fresca, a massa de água, os sólidos solúveis e os diâmetros transversal e ventral terem dado coeficientes de correlação com a massa seca maiores que o produto dos diâmetros, esse último apresenta a vantagem de ser uma medida mais confiável (Tabela 2). A massa fresca, massa de água e sólidos solúveis são muito sensíveis a variações climáticas (Castro Neto \& Reinhardt, 2003), e uma medida de produtos de diâmetros é sempre mais fidedigna que a avaliação individual de cada diâmetro. Assim, consideramos que o produto dos diâmetros dos frutos pode ser usado com segurança para determinar o crescimento da manga cv. Tommy Atkins, com a vantagem de ser um método não-destrutivo, baseado em medições fáceis e rápidas.

\section{CONCLUSÕES}

1. As mangas da cv. Tommy Atkins atingiram a maturidade aos 98 DAA, quando apresentavam a maior massa seca.

2. A massa seca foi o melhor indicador do estádio de desenvolvimento do fruto da mangueira cv. Tommy Atkins cultivada sob irrigação no Submédio São Francisco, considerando-se o seu alto coeficiente de determinação $\left(\mathrm{R}^{2}=\right.$ $0,99)$.

\section{AGRADECIMENTOS}

À Fundação Cearense de Apoio ao Desenvolvimento Científico e Tecnológico (FUNCAP), pelo suporte financeiro através do fornecimento de bolsa de pesquisa, à Fazenda Fruit Fort Agrícola Exportação Ltda., pelo apoio logístico, e à Embrapa Semi-Árido, pelo apoio laboratorial.

\section{REFERÊNCIAS}

ALBUQUERQUE, J. A. S. de; MEDINA, V. D.; MOUCO, M. A. do C. Indução floral. In: GENÚ, P. J. de C.; PINTO, A. C. de Q. A cultura da mangueira. Brasília: Embrapa Informação Tecnológica, 2002. cap. 13, p. 259-276.

ALMEIDA, C. O. de; CARDOSO, C. E. L.; SANTANA, M. do A. Comercialização. In: PEREIRA, M. E. C.; FONSECA, N.; SOUZA, F. V. D. (Ed.). Manga: o produtor pergunta, a Embrapa responde. Brasília: Embrapa Informação Tecnológica, 2005. cap. 15, p. 177-184. (Coleção 500 perguntas, 500 respostas).

AMORIM, T. B. F. Colheita e pós-colheita: manejo e conservação da manga. In: SÃO JOSÉ, A. R. (Org.). O agronegócio manga: produção e mercado. Vitória da Conquista: UESB/DFZ, 2002. p. 346-356. 1 CD-ROM.

AOAC - ASSOCIATION OF OFFICIAL ANALYTICAL CHEMISTS. Official methods of analysis. $16^{\text {th }}$ ed. Arlington: AOAC, 1995. $1141 \mathrm{p}$.

CASTRO NETO, M. T. de; REINHARDT, D. H. Relações entre parâmetros de crescimento do fruto de manga cv. Haden. Revista Brasileira de Fruticultura, Jaboticabal, v. 25, n. 1, p. 35-37, 2003.

CUNHA, G. A. P. da; SAMPAIO, J. M. M.; NASCIMENTO, A. S. do; SANTOS FILHO, H. P.; FONSÊCA, N. A cultura da manga. Brasília: Embrapa-SPI, 1994. 54 p. (Coleção Plantar, $10)$.

CUNHA, G. A. P. da; PINTO, A. C. de Q.; FERREIRA, F. R. Origem, dispersão, taxonomia e botânica. In: GENÚ, P. J. de C.; PINTO, A. C. de Q. A cultura da mangueira. Brasília: Embrapa Informação Tecnológica, 2002. cap. 2, p. 31-36.

DONADIO, L. C. Variedades de manga. In: São José, A. R. (Org.). O agronegócio manga: produção e mercado. Vitória da Conquista: UESB/DFZ, 2002. p. 119-126. 1 CD-ROM.

EMBRAPA. Dados meteorológicos da estação agrometeorológica de Bebedouro. Petrolina: Embrapa SemiÁrido, 2006. Disponível em: <http://www.cpatsa.embrapa.br/ serviços/ dadosmet/ceb-anual.html $>$. Acesso em: 22 jun. 2006. 
LEDERMAN, I. E.; BEZERRA, J. E. F. CARVALHO, P. S. de; ALVES, M. A.; SANTOS, V. F. dos. Determinação do ponto de colheita da manga cv. Tommy Atkins, para a região semiárida de Pernambuco. Revista Brasileira de Fruticultura, Cruz das Almas, v. 20, n. 2, p. 145-151, 1998.

LEITE, J. C. A.; GOUVEIA, J. P. G. de; ALMEIDA, F. de A. C.; SILVA, M. M. da; SILVA, R. G. da; PEDROZA, J. P. Caracterização física da manga variedade Tommy Atkins. In: SIMPÓSIO BRASILEIRODE PÓS-COLHEITADEFRUTOS TROPICAIS, 1., 2005, João Pessoa. Anais... João Pessoa: Embrapa/UFPB/UFS/SBF, 2005. 1 CD-ROM.

MORAIS, P. L. D. de. Maturidade para colheita e vida útil da manga 'Tommy Atkins' para o mercado europeu. 2001. $83 \mathrm{f}$. Dissertação (Mestrado em Agronomia)-Universidade Federal do Ceará, Fortaleza, 2001.

PINTO, A. C. de Q.; COSTA, J. G. da; SANTOS, C. A. F. Principais variedades. In: GENÚ, P. J. de C.; PINTO, A. C. de Q. A cultura da mangueira. Brasília: Embrapa Informação Tecnológica, 2002. cap. 5, p. 93-116.

SUBRAMANYAN, H.; SHANTHA, K.; PARPIA, H. A. B. Physiology and biochemistry of mango fruit. Advances in Food Research, San Diego, v. 21, p. 223-305, 1975.

TUCKER, G. A. Introduction. In: SEYMOUR, G. B.; TAYLOR, J. E.; TUCKER, G. A. Biochemistry of fruit ripening. London: Chapman \& Hall, 1993. p. 1-51. 